\title{
Surface-state emission enhancement in white-luminophor CdS nanocrystals using localized plasmon coupling
}

\author{
T. Ozel, ${ }^{1}$ I. M. Soganci, ${ }^{1}$ S. Nizamoglu, ${ }^{1}$ I. O. Huyal, ${ }^{1}$ E. Mutlugun, ${ }^{1}$ S. Sapra, ${ }^{2}$ N. Gaponik, ${ }^{2}$ A. Eychmüller, ${ }^{2}$ and \\ H. V. Demir ${ }^{1}$ \\ ${ }^{I}$ Devices and Sensors Group, Department of Physics, Department of Electrical and Electronics Engineering, Nanotechnology \\ Research Center, and Institute of Materials Science and Nanotechnology, Bilkent University, TR-06800, Ankara, Turkey \\ ${ }^{2}$ Physical Chemistry/Electrochemistry Group, Technische Universität Dresden, Bergstr. 66b, 01062 Dresden, Germany \\ E-mail: volkan@bilkent.edu.tr
}

There has been a great interest in the development of white nano-luminophors because of their potential use in solidstate lighting, which offers a significant level of reduction $(\sim 50 \%)$ in the global electrical energy consumption for lighting [1]. In the previous literature, magic-size CdSe nanocrystals (NCs) and Mn-doped CdS NCs have separately been shown to successfully generate white light emission, though with low quantum efficiencies of $<10 \%[2,3]$. In a recent work, the synthesis of trap-rich CdS nanocrystals with an average diameter of $2.4 \mathrm{~nm}$ has been reported with white surface-state emission, featuring relatively higher quantum efficiency ( $\sim 17 \%)$ [4]. These white nanoluminophor CdS NCs have a band-edge emission (from conduction band to valance band) at $405 \mathrm{~nm}$ and a broad surface-state emission (through mid-gap trap states) covering the visible spectrum. However, their surface-state emission is still 9 times weaker than their band-edge emission, which necessitates substantial enhancement of their surface-state emission for bright white light generation.

In this work, to make surface-state emission stronger than band-edge emission for the first time, we proposed and demonstrated plasmon coupling of the surface-state emission from the traps in these CdS nano-luminophors using localized plasmons. While the previous works of our groups and other focused only on enhancement of the bandedge emission between ground electronic e-h states from the conduction band to the valance band [5-6], the enhancement of surface-state emission through the mid-gap traps with plasmon coupling has not been achieved till date. To this end, we tuned localized plasmon resonance peak of our nano-Ag films to spectrally match the surfacestate emission peak of our trap-rich CdS NCs, and consequently demonstrated that the surface-state emission of these NC luminophors was significantly enhanced, while their band-edge emission was significantly suppressed at the same time. The ratio of the surface-state emission to the band-edge emission was improved by a factor of 6.8 as a result of strong plasmon coupling to the trap states. This corresponded to a giant enhancement factor of 12.7 folds in the ratio of the surface-state emission peak to the band-edge emission peak in the plasmon coupled film compared to the solution form. To the best of our knowledge, this is the first demonstration of plasmon-coupled surface-state emission using proximal metal nanostructures to make the surface state emission stronger than the band-edge emission in such white nano-luminophors.

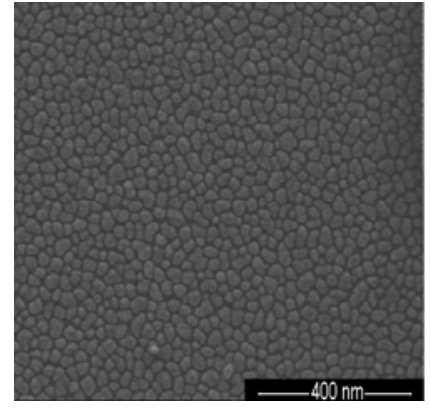

(a)

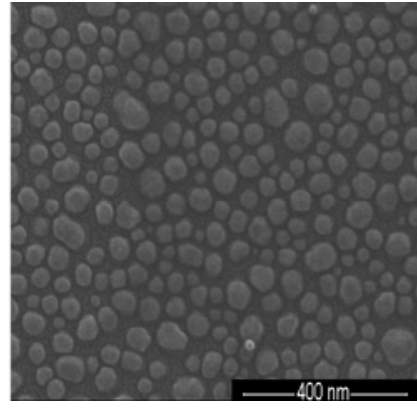

(b)

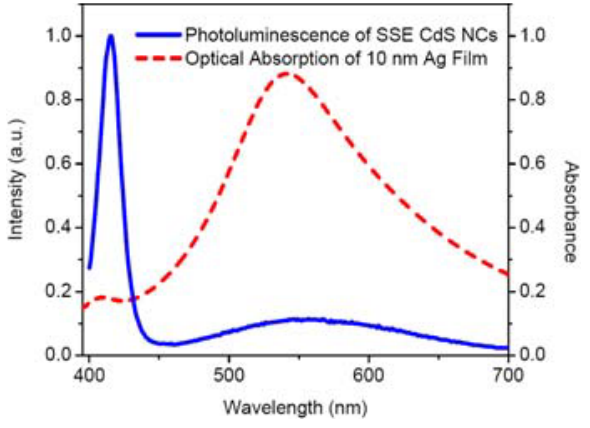

(c)

Figure 1. Scanning electron microscopy (SEM) images of our $10 \mathrm{~nm} \mathrm{Ag}$ nano-island film (a) before and (b) after annealing $\left(300{ }^{\circ} \mathrm{C}, 10 \mathrm{~min}\right.$.) to match (c) their optical absorption (dotted) with the photoluminescence of our CdS nanocrystal luminophors (solid).

We prepared metal nano-island films shown in scanning electron microscopy (SEM) images of Fig. 1(a)-(b) to provide localized plasmon resonance in the visible region. We fabricated Ag nano-island films with a height of 10 $\mathrm{nm}$ and varying lateral sizes of $25-75 \mathrm{~nm}$. We tuned the plasmonic resonance of our Ag nano-island films by annealing, which separated the islands from each other while making them bigger as depicted in Fig. 1(b). This resulted in red-shift of the plasmonic resonance wavelength. The optical absorption spectrum of our $10 \mathrm{~nm} \mathrm{Ag} \mathrm{nano-}$ island film depicted in dotted line of Fig. 1(c) was optimized to match the optical emission spectrum of surface-state 
emitting CdS NCs presented in the solid line of Fig. 1(c). We synthesized these CdS NCs using the earlier reported method [4]. During the synthesis, we intentionally introduced small-sized NCs to obtain a broad surface-state emission covering the entire visible spectrum. The resulting surface-state emission was found to be 8.9 weaker than the band-edge emission as given in solid line of Fig. 1(c). We prepared different solid samples, each to contain a 60 $\mathrm{nm}$ thick film of trap-rich CdS NCs in a PMMA matrix on Ag nano-island film deposited on a quartz substrate. For comparison, we used a negative control group that included an identical nanocrystal film placed directly on top of a quartz substrate (without nano-Ag). Fig. 2(a) compares the PL spectra of these samples under identical experimental conditions. We observed that the plasmonic nano-Ag suppressed the band-edge emission of CdS NCs, while simultaneously enhancing their surface-state emission. As a result of these dual interactions, the ratio of the surfacestate emission peak to the band-edge emission peak was increased by a factor of 6.8 in the film, yielding an enhancement factor of 12.7 for the plasmon coupled film sample compared to that in solution. In addition, with these many trap states coupled with plasmons, the total number of photons emitted at all wavelengths (including both the band-edge emission and the surface-state emission) was enhanced by $80 \%$. Fig. 2(b) and Fig. 2(c) show the pictures of these CdS NCs in solution (as synthesized) and in film (after plasmon coupling), respectively. Additionally, by investigating time-resolved fluorescence decays of these two films, we found out that, in the presence of nano-Ag, the trap-associated recombination became slower while the band-edge emission was faster as compared to the NCs film on quartz. This implies an enhanced efficiency of trap state population in the presence of the silver islands. This behavior is similar to the enhanced intersystem crossing rates from singlet and triplet excited states in aromatic molecules in the presence of heavy atoms. To understand the detailed energy transfer mechanism of these observed plasmon mediated electron transitions, further experiments are currently in progress in our groups.

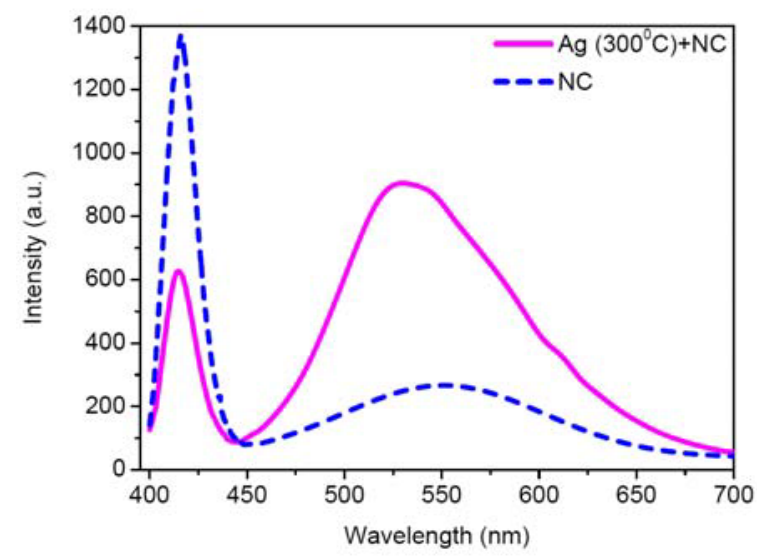

(a)

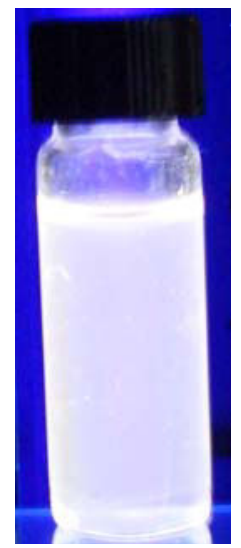

(b)

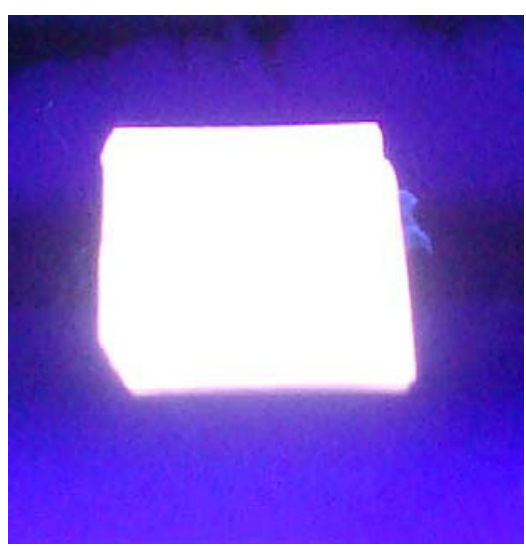

(c)

Figure 2. (a) Photoluminescence spectra of CdS NCs film alone (dotted) and when plasmon-coupled with a $10 \mathrm{~nm}$ thick Ag nano-island film (solid). (b) Picture of these NC luminophors in solution under UV lamp excitation. (c) Picture of the plasmon coupled NC luminophor film coupled with the silver nano-island film under UV light excitation.

In summary, we demonstrated plasmonic coupling of mid-gap trap states in trap-rich CdS NCs with proximal nano$\mathrm{Ag}$ films by spectrally matching their localized plasmon resonance wavelengths to the surface-state emission peak of these white nano-luminophors. We showed that, with special plasmonic engineering of these white nano-luminophor $\mathrm{NCs}$, the ratio of the surface-state emission in the visible to the band-edge emission was increased by more than 12 times, and the total number of photons emitted at all wavelengths was increased by $80 \%$. These results hold great promise for future NC-based solid-state lighting technology.

\section{Acknowledgments}

This work is supported by EU-PHOREMOST NoE 511616, Marie Curie EU-IRG MOON 021391 and TUBITAK 107E088, 107E297, 106E020, 104E114, 105E065, and 105E066. Also, HVD acknowledges additional support from Turkish Academy of Sciences (TUBA) Distinguished Young Scientist Award (GEBIP) and European Science Foundation (ESF) European Young Investigator Award (EURYI) Programs.

\section{References}

[1] The Promise of Solid State Lighting for General Illumination; Optoelectronics Industry Development Association: Washington, DC, 2001.

[2] M J Bowers, J R McBride, and S J Rosenthal, J. Am. Chem. Soc. 12715378 (2005).

[3] A Nag and D D Sarma, J. Phys. Chem. C 11113641 (2007).

[4] S Sapra, S Mayilo, T A Klar, A L Rogach, and J Feldmann, Adv. Mater. 19569 (2007).

[5] I M Soganci, S Nizamoglu, E Mutlugun, O Akin, and H V Demir, Opt. Express 1514289 (2007).

[6] O Kulakovich, N Strekal, A Yaroshevich, S Maskevich, S Gaponenko, I Nabiev, U Woggon, and M Artemyev, Nano Lett. 2 1449 (2002). 